\title{
NEW CHALLENGES IN COLLABORATIVE VIRTUAL FACTORY DESIGN
}

\author{
Stefano Mottura, Giampaolo Viganò, Luca Greci, Marco Sacco \\ Emanuele Carpanzano \\ Institute of Industrial Technologies and Automation \\ National Research Council, Milano, Italy \\ stefano.mottura@itia.cnr.it, giampaolo.vigano@itia.cnr.it, \\ luca.greci@itia.cnr.it,marco.sacco@itia.cnr.it, \\ emanuele.carpanzano@itia.cnr.it
}

\begin{abstract}
The present paper describes the results of recent and ongoing major European projects about the virtual factory design topic, highlighting in particular new challenges related to the networked collaborative design of factories layout and configuration. A pilot factory for the production of customized shoes is referred to throughout the paper as application example of the proposed methods and tools.
\end{abstract}

\section{INTRODUCTION}

In recent years a relevant change in factory conception, design and optimisation is ongoing, i.e. the use of digital design in many different aspects of a "production/factory/system": the intensive use of digital models and simulations (numerical and visual, also real-time), the growth of digital and virtual prototyping as tools supporting the evaluation and decision making process before the real product is released, are techniques and processes becoming more and more part of the life of a "factory product". In this paper, within the above context, a project, lead by ITIA - CNR, is presented, aimed to develop an application framework based on 3D-realtime-interactive solutions for the networked collaborative design of new factories layouts.

\section{PREVIOUS WORK}

The virtual factory, conceived as a system that can be digitally simulated under various points of view, has been carried on by ITIA in previous years (Böer 1996, Sacco 2000, Carpanzano 2004). The first effort on such a theme has been the ManuFuturing project where a deep analysis on the main aspects of a factory to be digitally represented has been executed. Discrete event simulation was used to simulate models of the production coupled with a $3 \mathrm{D}$ immersive virtual environment as a new interface for creating the production layout (see Figure 1). 


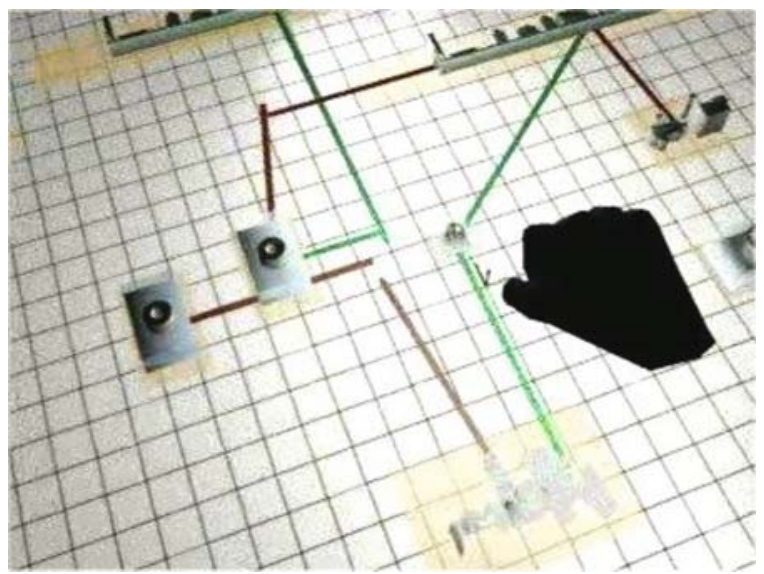

Figure 1. First example of virtual reality environment for factory layout design.

The virtual reality and advanced interfacing tools for editing the plant and/or factory layout has then been developed in the Modular Plant Architecture (MPA) Project, see also (Sacco 2004), where a more modular and enhanced designing environment was developed (see Figure 2 and Figure 3). In this new application the plant layout work was simulated by an external process simulator.

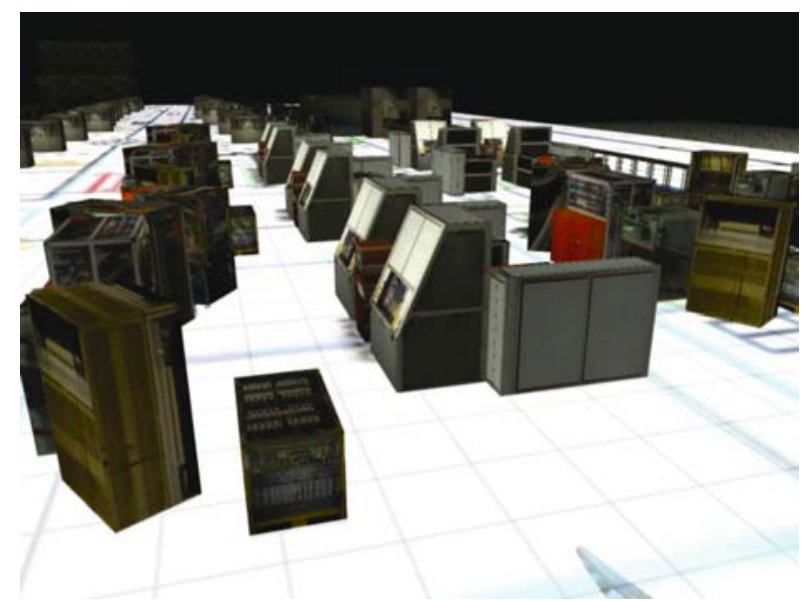

Figure 2. The virtual factory environment. 


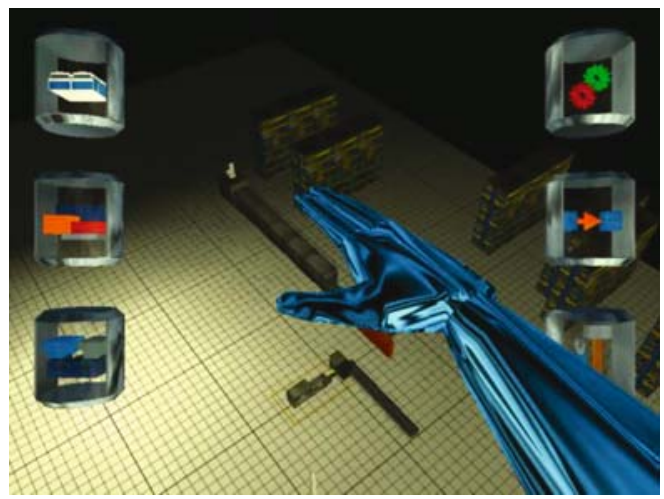

Figure 3. The user interface.

Another important step in the virtual factory design research was the simulation of the ITIA pilot factory for the customized shoes production in Vigevano, Italy (see also Sacco 2004, Carpanzano 2004). In this application the whole laboratory has been reproduced in 3D with a first-person navigation model (see Figure 4). Such an innovative factory allows consumers to order a personalized pair of shoes, different as for models, colours, materials and consumer's feet geometrical data. Thus, the main feature of the pilot factory is a high level of flexibility and reconfigurability. With the virtual environment the user can compose the production process by selecting the particular machines among the whole factory, as happens in real reconfiguration (see Figure 5). The movements of the machines and the flow of the semi-finished materials are simulated by an external process simulator. As shown in the figures, also the graphical quality of the environments has been enhanced, thanks to the rendering libraries developed at ITIA during last years.

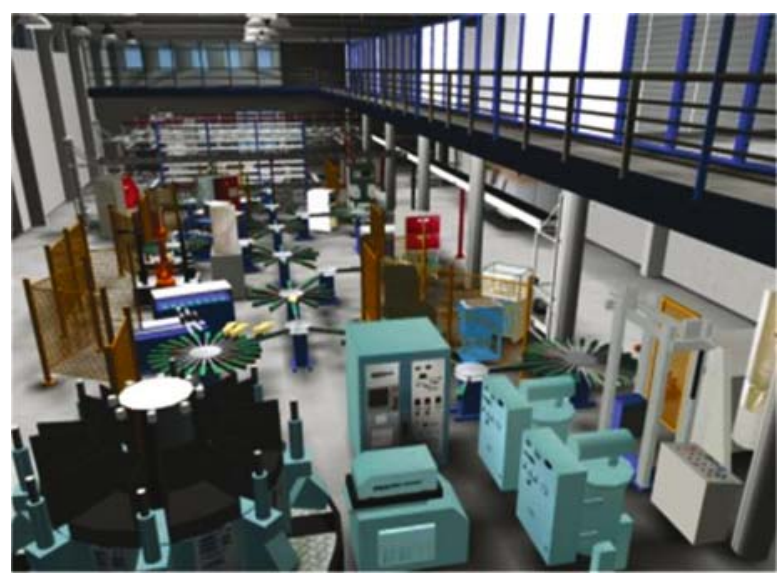

Figure 4. Virtual factory of the ITIA-CNR pilot factory in Vigevano, Italy. 


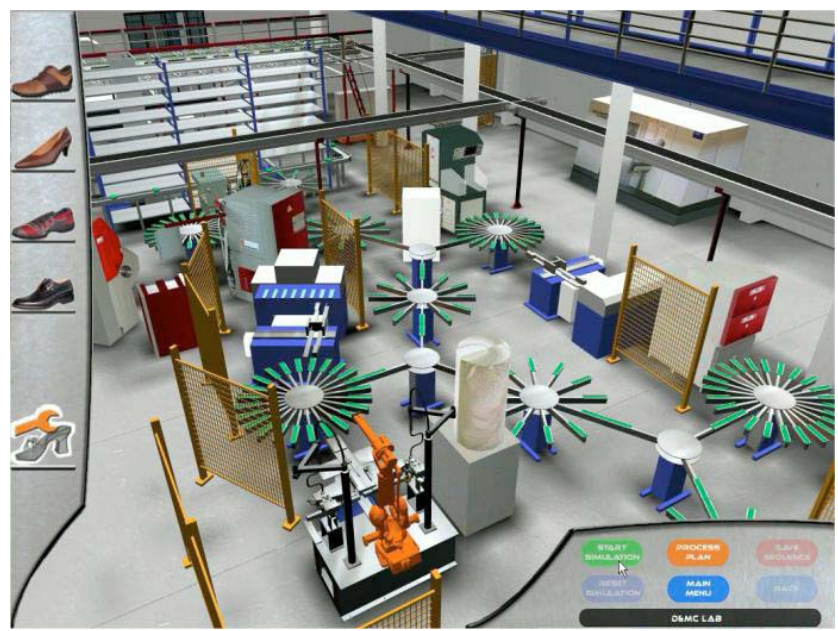

Figure 5. Production line configuration in the virtual factory environment.

Furthermore, the factory control system was developed through the support of digital facilities for the analysis, verification and optimisation of the automation functionalities, from the very first phases of the design life cycle (Carpanzano 2004, Carpanzano 2007). At the beginning of the design life cycle, the control and supervision system was specified by means of UML (Unified Modelling Language) diagrams; in particular use-case, class, sequence and state diagrams were used. The system architecture and functions were designed using the Function Block formalism defined by the IEC 61499 standard. The functional model obtained was analysed and optimized by means of discrete event simulations performed in the Simulink/Stateflow environment. When the functional modules had been verified, the control and supervision algorithms were developed using the SFC (Sequential Functional Chart) formalism. The SFC algorithms were analysed through closed loop dynamic simulations in the ISaGRAF environment in order to verify their correctness and to optimize their performance (Figure 6). In order to perform the closed loop simulations, simplified models of the plant devices have been represented by means of suitable SFC modules and data structures. Moreover, to simplify the analysis of the simulation results, a dedicated simple 2D graphic animation has been realized in ISaGRAF Simulations have been performed by considering typical operating conditions, i.e. typical production orders, and the results obtained show that the system is deadlock free and that the plant is well balanced, i.e. its resources are all used effectively. After the verification and optimization of the automation functions and algorithms the corresponding code was generated and implemented on the target industrial devices. Such simulation-based analysis techniques reduced the plant rump-up times and costs and improved overall system performance (Carpanzano 2007). 


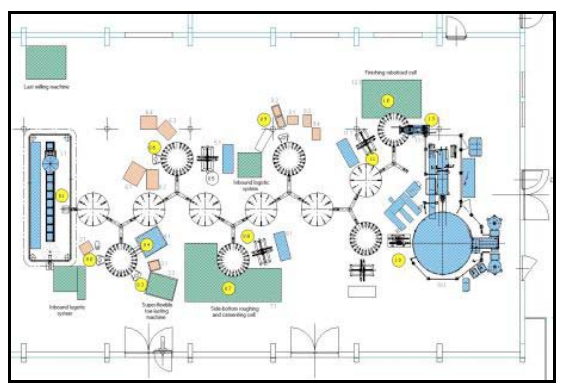

(a)

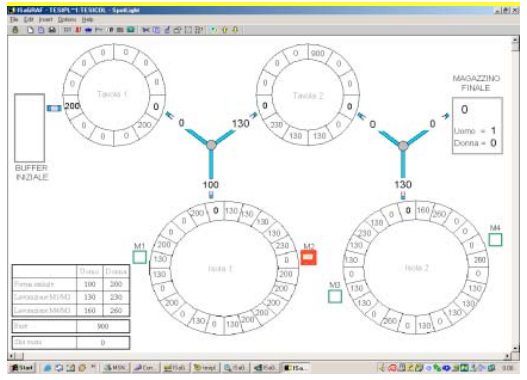

(b)

Figure 6. Layout of the Plant (a ) and Simulation Graphical Interface (b).

\section{THE DIFAC PROJECT}

Currently, a new challenge in digital factory design is being faced: the virtual factory design within collaborative real-time networks. The idea is to invest the experiences of the past and ongoing researches by adding the feature of an environment for factory layout design in a shared networked framework. The presented ongoing research is part of the DiFac Project (www.difac.net).

The framework will be composed by a set of distributed personal computer (PC) workstations, on the PCs the factory being designed is visualized simultaneously by all different users. Just with this capability the users become participants of a networked meeting, by sharing the same virtual environment: they can interactively share the subject of their discussion and their work. The shared virtual environment allows to identify a PC as server and as leader of operations, and the other PCs as clients and active participants. The users acting on the server can manage and manipulate the virtual environment layout (that can be for example a line, a process or a whole factory) while the clients observe in real-time the proposed design and may suggest their modifications. While clients have the opportunity to modify the virtual layout, only the server has the rights to confirm and store permanently the work done during the meeting and all the related data. In this context, the set of data fields that describe a factory layout ( $3 \mathrm{~d}$ models, textures, state of virtual objects, data of simulations and so on) will be called a project.

\subsection{System architecture}

Projects are stored in a database on the server and are composed by several data fields: name, identifier, original layout description, alternative layout descriptions, references to data of the virtual environment as $3 \mathrm{~d}$ models, textures, simulation conditions and results. The core common to both server application and client application supports the following operations:

- visualize the list of available projects (the virtual layouts in progress),

- access to the representative data fields of a specific project,

- $\quad l o a d$ the $3 \mathrm{~d}$ virtual environment that represents a specific layout,

- $\quad$ interact with a specific layout: navigate, select and edit virtual entities.

The server retrieves data directly from the database and shares them over the network with all connected clients. So, while the server has a direct access to the database, the 
clients can deal with projects by interfacing though the server (see Figure 7). Let's remind here that the server is not intended merely as a database server.

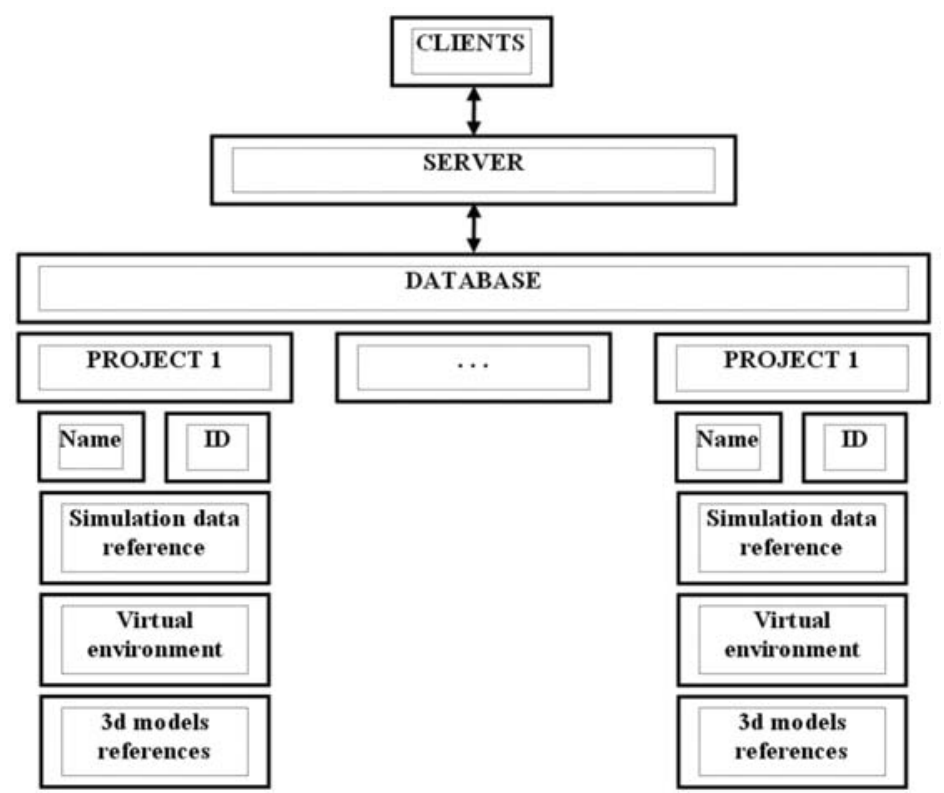

Figure 7. The server is the interface to access the projects database.

\subsection{Session operation model}

The execution of a collaborative virtual factory layout editing session is quite inspired to networked arcade real-time $3 \mathrm{~d}$ games, like cars or motorbikes races. People join the game and every player sees who is connected; then, through dedicated communication channels (typically a chat embedded in the game) all the players agree on a specific circuit where to play the race (or, alternatively, the server imposes all its own decision). Afterwards, the game starts and all the players see and share interactively in real-time the same $3 \mathrm{~d}$ race. With respect to this example, people join the shared virtual factory application and every person can see who is connected and, through dedicated communications (embedded chat, previous email and so on) all the people agree on a specific project to edit from a set of available project information broadcasted by the server. Then, the collaborative session starts, and all people can modify and propose their suggestions by acting in the same virtual environment.

With respect to the example of the cars race game (where every player runs by himself), in a project session all the players operate on the same objects and data. To do this in a coherent way, like in real human meetings, a player, before doing any operation in the virtual environment, should select the object to manage. The act of selecting locks the object for that player that has exclusive access to the object itself. The other players can see in real-time what it is happening on that object (or set of objects) in the virtual environment.

The behaviour of this logic is managed and maintained by the server application. When a client selects an object it sends to the server a request of exclusive ownership, the server receives it and checks if it is possible and then sends back the to the client the answer (see Figure 8). On the other side, relevant operations that can be done locally, and 
in an independent way, are the navigation in the virtual environment and project database consulting (always through the transparent interface of the server).

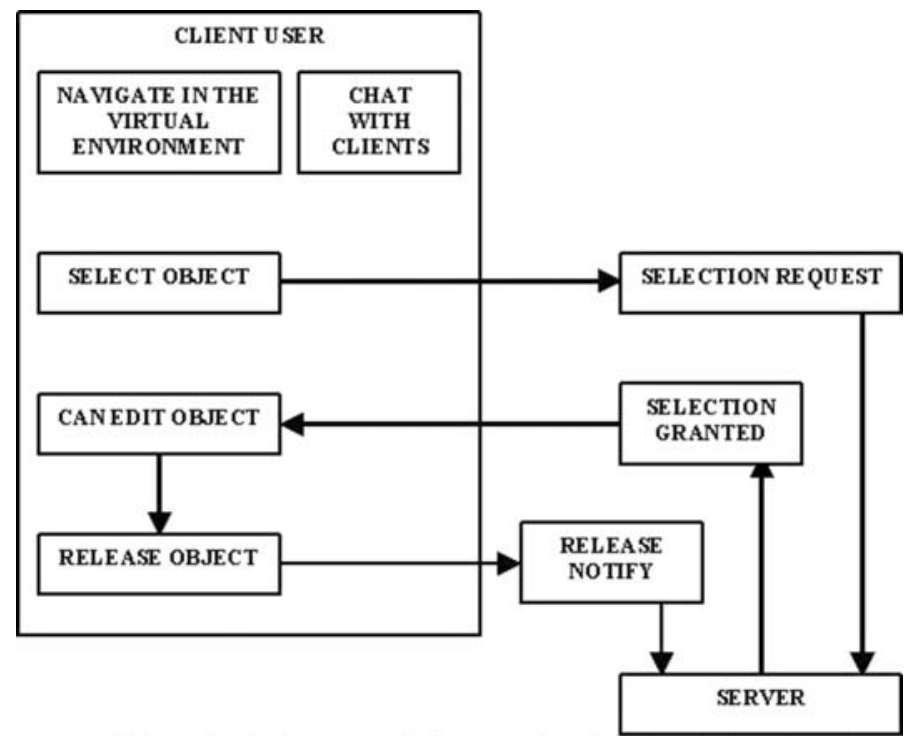

Figure 8. Collaborative logic protocol for accessing the shared virtual environment.

Once that a virtual layout is accepted by all participants, it can be saved into the database by the server side, thus it will be available for further editing for next sessions.

The selection mechanism is not the only logical operation managed by the server but it is the important one for managing an entity of the shared virtual environment. The other important behaviours handled by the server are:

- manage accesses to the underlying database of projects,

- manage the participations of a session: join/abandon; broadcast clients identificative information,

- manage potential clients limitations in the rights of editing virtual entities.

\section{CONCLUSIONS}

The framework under development presented in this paper is an example of integration and use of enabling technologies and techniques aimed to a collaborative virtual environment for virtual factory layout editing and design. The described platform adopts networking software techniques, virtual reality techniques, as well as collaborative means, and integrates them in a software unique application that, in the context of the DiFac Project, is aimed to the factory layout, but its potential concept obviously can be applied also in the important field of the collaborative networked product design and evaluation, where the "product" is just the object to be pushed into the market. Another relevant point is that, as for software modules and functionalities development, ITIA is managing and storing the functionalities in a programming library, that is an extension of existing ITIA's library for managing $3 \mathrm{~d}$ real-time graphics, so that a base platform will be available and reusable for developing further shared virtual environments. 


\subsection{Acknowledgments}

The work and research results described in this paper have been carried on by ITIA CNR in the context of the following major projects:

Integrated Programme $N^{\circ} 507378$ CEC-Made-Shoe "Custom, environment and comfort made shoe", 6 FP Priority IST- NMP (Manufacturing, Products and Service Engineering 2010);

EUROShoE Project, Contract G1RD-CT-2000-00343, Project N GRD1-2000-25761; 5 FP Growth;

DiFac Project, Contract $N^{\circ}$ 035079, Digital Factory for Human-Oriented Production System, 6FP STREP.

\section{REFERENCES}

1. DiFac Project website: www.difac.net.

2. Sacco M, Mottura S, Greci L, Viganò G, Böer CR. "Experiences in virtual factory prototype: modular plants design and simulation". IFAC-MIM 2004, Conference on Manufacturing, Modelling, Management and Control, 2004.

3. Sacco M, Mottura S, Viganò G, Avai A, Böer CR. "Tools for the innovation: virtual reality and discrete events simulation to build the 2000 Factory". Proceedings of AMSMA 2000, pp. 458-462.

4. Böer CR, Jovane F. "Towards a new model of sustainable production: ManuFuturing". CIRP Annals STC O, 05/01/1996, pp. 415.

5. Carpanzano E. Cataldo A. Innovating Shoe Manufacturing using Advanced Simulation Techniques. ERCIM News. Special Theme: Industrial Diagnosis, Planning and Simulation 2004; N 56: 22-23.

6. Carpanzano E., Jovane F. Advanced Automation Solutions for Future Adaptive Factories. Annals of the CIRP Vol. 56/1/2007. 